\title{
End-Stage Complications of Diabetic Neuropathy: Foot Ulceration
}

\author{
Andrew J.M. Boulton
}

\begin{abstract}
Diabetic polyneuropathy is a major complication of diabetes mellitus that frequently leads to foot ulceration. Because of economic impact of foot ulceration and subsequent amputation, major initiatives are under way in the United States and the United Kingdom to reduce their incidence by up to $50 \%$. Although diabetic ulcers may have an ischemic and neuropathic component, the majority are neuropathic. Results from studies have shown a strong association between the neuropathy and the subsequent development of foot ulcers. However, other predisposing factors such as high foot pressures, inappropriate footwear, and other extrinsic pressure usually must be present, together with neuropathy, to cause foot ulcers. Therefore, the challenge to the physician is to identify the patient at risk and intervene so as to educate and prevent the occurrence of foot ulcers.
\end{abstract}

\begin{abstract}
Résumé: Complication ultime de la neuropathie diabétique: L'ulcère du pied. La neuropathie diabétique est une complication majeure du diabète qui conduit souvent à l'ulcère du pied. A cause de l'impact économique de l'ulcération du pied et d'une amputation éventuelle, les Ètats-Unis et la Grande Bretagne ont pris des mesures importantes pour réduire son incidence d'au moins $50 \%$. Bien que les ulcères diabétiques puissent avoir une composante ischémique et neuropathique, la majorité sont d'origine neuropathique. Des études ont démontré qu iil existe une association entre la neuropathie et le développement subséquent de l'ulcère du pied. Cependant, d'autres facteurs prédisposants tels des pressions élevées au niveau de pied, des chaussures inadéquates ainsi que d’autres sources de pression extrinsèque doivent généralement être concomitants avec la neuropathie pour causer un ulcère du pied. Le défi pour le clinicien est donc d'identifier le patient à risque et d'intervenir pour éduquer son patient et prévenir l'ulcère du pied.
\end{abstract}

Can. J. Neurol. Sci. 1994; 21: Suppl. 4-S18-S22

One of the most common long-term complications of diabetes mellitus is diabetic polyneuropathy. Neuropathy rarely occurs within the first five years after the onset of type 1 diabetes, but by 25 years, almost $50 \%$ of diabetic patients have some signs of neuropathy and are often affected by loss of sensation in the lower extremities.' Such patients are at risk for chronic foot ulcers and, ultimately, lower extremity amputation. The focus of this presentation is not only to show that neuropathy causes foot ulceration but also to emphasize the economic importance of foot ulceration and amputation in diabetes.

\section{EPIDEMIOLOGY AND ECONOMIC IMPACT}

Lower extremity ulceration is a common complication of diabetes. A 1991 report from the Centers for Disease Control in Atlanta showed that the highest risk of major complications was for leg and foot ulceration, at $3.1 \%$ per year, while amputation was the third most common. The risk of other end-stage microvascular complications, including blindness from retinopathy and end-stage renal disease secondary to nephropathy, was only $0.1 \%$ per year. In the United Kingdom, diabetes ranks as the second most important cause of amputation accounting for $25 \%$ of major lower extremity amputations compared with $9 \%$ for trauma.
In a recent large, multicenter study of diabetic neuropathy in the United Kingdom, the risk of foot ulceration was assessed in 6,487 patients attending 120 diabetes clinics. ${ }^{2}$ The definition of "risk of foot ulceration" was a moderate or severe neuropathy disability score and/or two or more absent foot pulses. Under those criteria, $25 \%$ of patients were potentially at risk of foot ulceration. As expected, the risk increased with age, male gender, type 2 diabetes, and duration of diabetes. Foot ulcers were present in $2.2 \%$ of patients, and $2.5 \%$ were amputees.

The overall five-year mortality rate after amputation in all patients is $50 \%$, but in diabetic patients it is $70 \%$. In 1990, the European group of the World Health Organization met in St. Vincent, Italy, to develop five-year goals for reducing morbidity from diabetic complications. ${ }^{3}$ One goal was to reduce the rate of amputation due to diabetes by $50 \%$ within five years. Thus, the responsibility of the diabetologist is to identify patients at risk for foot problems and to prevent progression to more serious complications.

From The Royal Infirmary, University of Manchester, Manchester, England. Reprint requests to: Andrew J.M. Boulton, MD. FRCP. Department of Medicine, The Royal Infirmary. The University of Manchester. Manchester. England M139WL 
Examination of the economic costs of diabetic complications reveals that diabetic foot problems are responsible for $20 \%$ of all diabetic admissions to the hospital in both the United Kingdom and the United States. Prolonged hospitalization is often required: the mean duration of hospitalization for amputation in one study was 27 days (AJM Boulton, 1985, unpublished information). The American Diabetes Association has proposed that it should be possible to reduce the rate of amputation due to diabetes by at least $50 \%$. The cost of major leg amputations in the United Kingdom was about $£ 13.5$ million in 1985, or around Can\$27 million.

\section{Pathogenesis}

The pathogenesis of diabetic foot ulceration is complex and may be attributed to two primary etiologies (Figure 1): ${ }^{4}$ Largevessel obstructive peripheral vascular disease occurs in many patients and may be further aggravated by hyperlipidemia and cigarette smoking; it leads to foot ischemia and can directly cause ischemic foot ulceration. The second etiology-diabetic neuropathy-can be subdivided into somatic dysfunction and autonomic dysfunction. Somatic neuropathy leads to decreased pain sensation in the late stages, to decreased joint position sensation, and to small-muscle wasting of the foot. The patient with autonomic neuropathy experiences decreased skin sweating and dry skin, which combined with high foot pressures leads to callus formation and fissures under the feet. Foot pressure abnormalities occurs under the diabetic foot, and these are exacerbated by abnormalities of proprioception and muscle function, limitation of joint mobility, and other orthopedic problems. Loss of pain and sensation occur in the presence of sensory neuropathy, and the patient continues to use the foot despite the presence of a foot ulcer. Poor blood supply from small- and large-vessel disease leads to altered blood flow, and the ultimate outcome may be an ulcerated infected foot, which may on occasion lead to amputation.

Most foot ulcers have neuropathic and ischemic components, but purely ischemic ulcers and purely neuropathic ulcers also are recognized. In a 1974 animal study from Poland, somatic and sympathetic nerves to the hind limb of dogs were transected,

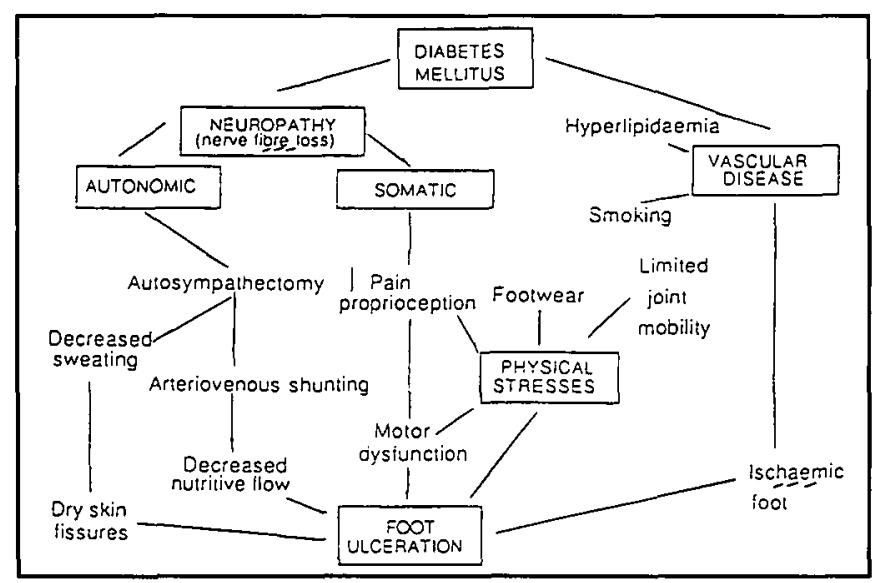

Figure 1:. Pathogenesis of foot ulceration in the patient with diabetes mellitus (Boulton, 1991. with permission). and the animals were followed for the development of foot ulcers. ${ }^{5}$ Ulcer formation was preceded by the opening of arteriovenous shunts, which was a manifestation of autonomic dysfunction after sympathectomy. Evidence of arteriovenous shunting has also been deomonstrated in diabetic patients with peripheral neuropathy; this was confirmed by the presence of increased venous oxygenation and also utrasound evidence of shunting using non-invasive measurements. ${ }^{6.7}$ The presence of this "auto sympathectomy" also leads to reduced sweating in the feet and consequent dry skin which cracks and fissures; together these form a further pathogenetic mechanism for insensitive foot ulceration. ${ }^{8}$

A cross-sectional study of diabetic patients with foot ulcers was undertaken at King's College Hospital in London. ${ }^{9}$ Of the first 239 patients assessed in the diabetic foot clinic, $62 \%$ had primarily neuropathic ulcers, $25 \%$ had mixed neuroischemic ulcers, and $13 \%$ had purely ischemic ulcers. Among patients studied for more than two years in the diabetic foot clinic at the Manchester Royal Infirmary, neuropathic ulcers were found in $45 \%$; mixed, in $39 \%$; and purely ischemic, in only $9 \% .{ }^{10}$ Therefore, neuropathy plays a major etiopathogenic role in the development of foot ulcers.

Ischemic peripheral vascular disease is a factor in diabetic foot ulcers, but peripheral neuropathy represents the most important etiology. A group of patients presenting with new foot ulcer in a diabetic foot clinic were compared with matched diabetic controls with no evidence of foot ulceration." Vibration perception threshold (VPT) was measured over the hallux using a handheld biothesiometer, which is a simple, semiquantitative measure of vibration perception. A strong association was noted between foot ulceration and VPT (Table).

A prospective study was undertaken in 489 patients in a diabetes clinic at the Manchester Royal Infirmary to determine the association between neuropathy and foot ulceration. ${ }^{2}$ Patients without a history of previous ulceration or peripheral vascular disease were evaluated and grouped according to baseline VPT. They were given the usual education about foot care and were followed during the next 3.5 to 4 years. Figure 2 shows the incidence of foot ulcers during the follow-up period according to VPT. In the group with normal VPT, the risk of foot ulceration was $0.7 \%$ per year. Those patients with a VPT $>25$ volts, which indicates the presence of neuropathy, had a sevenfold greater risk of developing a neuropathic foot ulcer during the next 3.5 years. These results provide clear evidence of a direct causal link between abnormalities of peripheral nerve function and the risk of foot ulceration.

In patients with active foot ulceration, positive, painful symptoms of neuropathy are unusual, but most patients give a

Table. Association between vibration perception threshold (VPT) and foot ulceration in diabetic patients with neuropathy.

\begin{tabular}{cccc}
\hline VPT & $\begin{array}{c}\text { Ulcer Present } \\
(\mathbf{n}=\mathbf{8 6})\end{array}$ & $\begin{array}{c}\text { Ulcer Absent } \\
(\mathbf{n}=\mathbf{4 9})\end{array}$ & Odds Ratio \\
\hline$<25$ & 11 & 30 & - \\
$25-32$ & 9 & 7 & 3.51 \\
$33-42$ & 16 & 6 & 7.27 \\
$>42$ & 50 & 6 & 22.73
\end{tabular}

(adapted from Boulton et al., 1986 11 , with permission). 


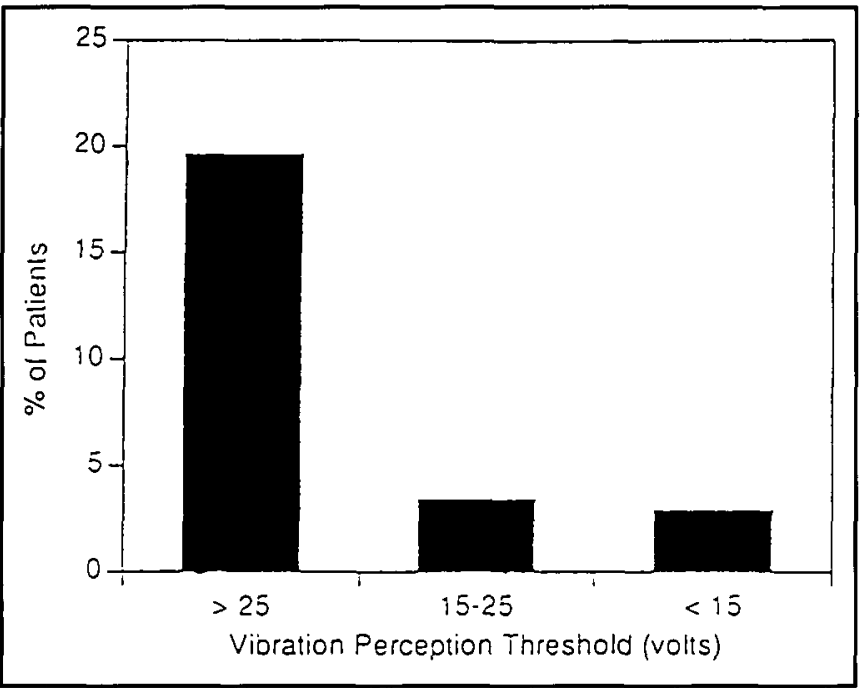

Figure 2: Incidence of foot ulceration among diabetic patients, assessed by vibration perception threshold (Young et al. ${ }^{2}$, with permission).

past history of neuropathic symptoms. The relationship between autonomic dysfunction and foot ulcers was studied in patients with symptomatic and asymptomatic neuropathy. ${ }^{13}$ Electrophysiological and autonomic function tests were conducted in diabetic patients without symptoms of neuropathy, with newly developed painful neuropathy, with chronic painful neuropathy, and with asymptomatic neuropathy with recurrent foot ulcers. Electrophysiological and cardiovascular autonomic function tests were abnormal in symptomatic patients and those with foot ulcers. However, abnormalities in autonomic function were equally present in patients with painful neuropathy, whereas electrophysiological abnormalities were greater in patients with foot ulceration.

To summarize, purely ischemic ulcers are rare, and, rather neuropathy can be implicated as the cause of $90 \%$ of foot ulcers. Patients with a warm but insensitive foot are at risk for neuropathic foot ulceration. Such patients often have a false sense of security because of the absence of pain and the loss of sensation. They believe that ulcers are caused by circulation problems, and thus a major challenge is to educate patients that the loss of sensation is a major risk factor for foot ulcers.

\section{MECHANISMS OF Ul.CERATION}

What is the pathogenesis of foot ulcers? In the late stages, sensory neuropathy leads to loss of pain and sensation, and motor neuropathy leads to an imbalance between the flexor and extensor muscles in the small muscles in the feet, which causes the loss of protective soft tissues. The fat pads normally located under the metatarsal heads migrate forward, which leads to changes in the forces acting under the feet. This can be measured as vertical forces, which are perpendicular to the surface, or shear forces, which are tangential to the actual surface.

The mechanisms causing foot ulcers in the insensitive foot can be appreciated by remembering that pressure equals force divided by area. One mechanism is constant maintained pressure, which leads to ischemic necrosis. An example is the patient who, because of insensitivity, buys a pair of shoes that are too small. Because such patients need more pressure per unit area to stimulate any remaining nerve fibers, they do not feel the normal discomfort, and thus they wear the shoes for several hours, causing ischemic necrosis at sites of high pressure, such as the lateral edge of the fifth or the first metatarsal head. Another mechanism is represented by the sustaining of high pressure on a small surface area for a short period of time, such as standing on a thumbtack, which leads to immediate injury. The most common mechanism is probably repetitive moderate stress: the insensitive foot exposed to high foot pressures and repetitive stress leads to skin breakdown.

In humans, semiquantitative measures for assessing pressures of the foot include the Harris-Beath mat, which is a piece of paper sitting on a ridged, foam-rubber mat that sits atop an inked pad with a piece of paper underneath. ${ }^{14}$ The more contact between the foot and the ridges, the greater the imprint onto the paper. A more objective assessment is the optical pedobarograph, which assesses pressures and loads under the foot during standing and walking. ${ }^{15}$ It yields a pressure-time curve $\left(\mathrm{kg} / \mathrm{cm}^{2}\right)$ of specific areas of the foot during standing and walking.

A prospective study was conducted to evaluate the effect of high, dynamic foot pressures on foot ulceration in the susceptible diabetic foot. ${ }^{16} \mathrm{~A}$ series of 86 diabetic patients including 58 with neuropathy at baseline were followed for 2.5 years. Dynamic foot pressures were assessed at baseline and at a follow-up visit. In the nondiabetic control group, foot pressures remained unchanged after a 35 -month follow-up. Among patients with high plantar pressures, $35 \%$ developed foot tilcers, and in the subgroup with neuropathy at baseline and high foot pressures, $45 \%$ developed ulcers. In contrast, no patient with normal foot pressures developed a foot ulcer (Figure 3). Therefore, high plantar foot pressures were strongly predictive of foot ulcers, especially in the presence of neuropathy. Highpressure sites can change over a short period of time, and techniques to reduce high pressures can reduce the high incidence of foot ulcers in the at-risk neuropathic foot.

To summarize, the neuropathic foot with good circulation does not spontaneously ulcerate but ulcerates only in the presence of some intrinsic or extrinsic trauma. Patients with neuropathy and

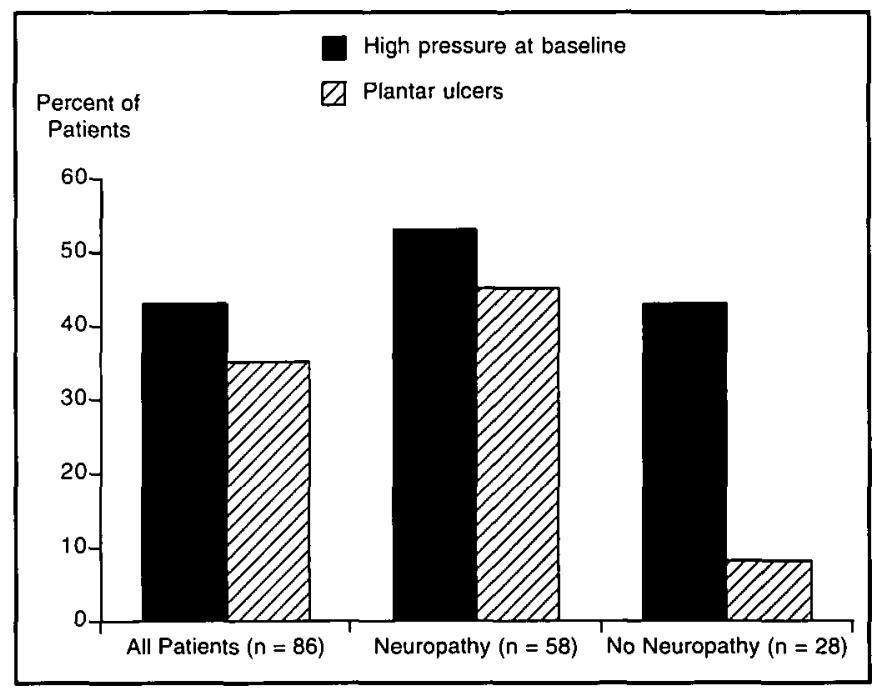

Figure 3: Incidence of high foot pressures and subsequent occurrence of plantar foot ulcers in diabetic patient with and without diabetic neuropathy (Veves et al., 1992', with permission). 
intrinsic ulcers often have very high foot pressures, and repetitive pressure during walking will lead to skin breakdown. Ulcers may develop in patients with neuropathy when their feet are exposed to extrinsic pressures, such as standing on a nail, wearing undersized shoes, or sustaining thermal burns. In the purely neuropathic ulcer, patients with complete insensitivity put their feet under extrinsic pressure with ill-fitting footwear, for example, thus leading to an extrinsic ulcer. Patients with neuropathy and limited joint mobility have very stiff joints, which contributes to high foot pressures. ${ }^{17}$ Such patients develop abnormal intrinsic foot pressures, and the repetitive pressure from day-today walking may lead to skin breakdown and ulceration.

What about the route that leads to lower extremity amputation? A study was done of 80 consecutive amputations performed over 30 months at the Seattle Veterans Administration Center. ${ }^{18}$ Approximately $60 \%$ of amputations were attributable to neuropathy, and $86 \%$ were potentially preventable. A number of causes of amputation were identified that individually did not lead to amputation, but together formed a sufficient insult resulting in amputation. For example, a neuropathic foot alone does not lead to ulceration or amputation, but if the neuropathic foot is traumatized by stepping on a nail and the patient continues to walk on the nail because of the absence of sensation, the combination of neuropathy, trauma, and ulceration causes a skin lesion. An infection ensues; the foot becomes edematous, which impairs arterial inflow; and gangrene develops. Therefore, the combination of neuropathy, trauma, ulceration, faulty healing, and gangrene provides a sufficient cause for amputation.

\section{Management}

As physicians, our challenge is to identify patients at high risk for foot ulceration and to concentrate educational and prevention efforts in this group. Clearly, patients with peripheral neuropathy and vascular disease are at risk, and the patients most at risk are those with previous foot ulcers and deformities of the foot. Our approach to the diabetic foot is PITS, which stands for prevention, by $i$ dentifying those at risk, providing an appropriate treatment, and the service for education.

A multidisciplinary approach to the diabetic foot, involving the podiatrist, diabetologist, orthotist, specialist nurse, and surgeon, is optimal. 19. These are the core individuals of the team and should form the minimum requirement in most centres. Additional personnel such as dermatologists, infectious disease specialists, rehabilitation physicians, plastic surgeons, and others may be added at certain centres if such individuals have an interest in the diabetic foot. The implementation of a diabetic foot service, which emphasizes the multidisciplinary approach to identification of foot ulcers and emphasizes patient education, has reduced the amputation rate by $43 \%$ in three years. ${ }^{9} \mathrm{~A}$ specialist nurse works only with diabetic foot problems, including answering questions on the telephone. Studies have shown that patients are more likely to call with questions if they know that a nurse will answer the phone rather than a physician.

In the diabetes clinic at the Manchester Royal Infirmary, a management algorithm is used for each new patient with diabetes, and at least annually thereafter. The patient is questioned and examined for any risk factors for foot ulceration, including neuropathy, vascular disease, and previous ulcers. In the absence of risk factors, patients receive general advice on nail care, podiatry, hygiene, and footwear that is appropriate to everyone, irrespective of diabetes. Patients who have insensitive feet or vascular problems need foot care education, regular podiatry, special footwear and hosiery, more frequent visits with the physician, and regular reinforcement of education. Patients at risk for foot ulcers receive more specific educational literature about foot care.

The principle at work in the treatment of the insensitive foot ulcer is removal of pressure. For instance, simply removing callus reduces abnormally high foot pressures by $35 \%$ to $40 \% .^{20}$ The foot can be protected from high foot pressures by the use of appropriate insoles, hosiery, and shoes. ${ }^{21-24}$ For example, the Scotch cast boot removes the patient's ability to weight-bear over the ulcer. Special shoes are very important, and the extra depth of the shoe is used for most patients with high-risk feet. Expensive, custom-designed shoes are usually needed only by patients with Charcot deformity or in other special circumstances. Special socks have an advantage over insoles, because the shoe becomes too small with the insole, producing a dorsal ulcer. Socks with special padding under high-pressure areas protect all around the foot and are generally accepted by patients. Some studies were done to examine ways in which hosiery can protect the high-risk, insensitive foot. ${ }^{22}$ Using the pedobarograph, special sports hosiery was shown to provide significant relief of pressure under the neuropathic foot, which was suṣtained for up to six months of regular usage.

As Voltaire said, in 1756, "One day, everything will be well; that is our hope. Everything is fine today; that is our illusion." That was certainly true about the diabetic foot until recently. Now, there are increasing interest in and realization of the sheer cost of diabetic foot problems to health economics. An increasing number of foot clinics are available, and the hope is that a reduction in large extremity amputation will be realized. The risk factors for foot ulcers and their interaction have been described. It is essential to identify patients at risk for foot ulcers and to reduce the chances of the most feared of all diabetic complications, that is, a major lower limb amputation.

\section{REFERENCES}

1. Pirart J. Diabetes mellitus and its degenerative complications: a prospective study of 4,400 patients between 1947 and 1973 . Diabetes Care 1978; 1 : 168-188, 252-263.

2. Young MJ, Boulton AJM, Macleod AF, Williams DRR, Sonksen $\mathrm{PH}$. A multicentre study of the prevalence of diabetic peripheral neuropathy in the United Kingdom hospital clinic population. Diabetologia 1993; 36: 150-154.

3. Krans HMJ, Porta M, Keen $H$. Diabetes care and research in Europe: the St. Vincent Declaration Action ProgrammeImplementation Document. Giornale Ital Diabetologia 1992: 12 (Suppl 2).

4. Boulton AJM. Clinical presentation and management of diabetic neuropathy and foot ulceration. Diabetic Med 1991; 8 (Suppl): S52-S57.

5. Nielubowicz J, Borkowski M, Baraniewski H. Opening of arteriovenous anastomoses and trophic ulcer formation after peripheral nerve injury. J Cardiovasc Surg 1974; 15: 636-646.

6. Boulton AJM, Scarpello JHB, Ward JD. Venous oxygenation in the diabetic neuropathic foot. Evidence of arteriovenous shunting? Diabetologia 1982; $22: 6-8$

7. Edmonds ME, Roberts VC, Watkins BJ. Blood flow in the diabetic neuropathic foot. Diabetologia 1982; 22: 9-15.

8. Boulton AJM. The diabetic foot: neuropathic and aetiology. Diabetic Med 1990; 7: 852-858.

9. Edmonds ME, Blundell MP, Morris ME, et al. Improved survival of the diabetic foot: the role of a specialized foot clinic. Q J Med $1986 ; 60: 763-771$. 
10. Thomson FJ, Veves A, Ashe $\mathrm{H}$, et al. A team approach to diabetic foot care. The Manchester experience. Foot 1991; 1: 75-82.

11. Boulton AJM, Kubrusly DB, Bowker JH, et al. Impaired vibratory perception and diabetic foot ulceration. Diabetic Med 1986; 3 : 335-337.

12. Young MJ, Breddy JL, Veves A, Boulton AJM. The use of vibration perception thresholds to predict diabetic neuropathic foot ulceration: a prospective study. Diabetes Care 1994; 17: 557-561.

13. Young RJ, Zhou YQ, Rodriguez E, et al. Variable relationship between peripheral somatic and autonomic neuropathy in patients with different syndromes of diabetic polyneuropathy. Diabetes 1986; 35: 192-197.

14. Boulton AJM. The importance of abnormal foot pressure and gait in the causation of foot ulceration. In: Bakker K, Kruseman ACN, eds. The Diabetic Foot. Amsterdam; Excerpta Medica; 1991: 3550.

15. Boulton AJM, Betts RP, Franks CI, Ward JD, Duckworth T. The natural history of foot pressure abnormalities in neuropathic diabetic subjects. Diabetes Res 1987; 5: 73-77.

16. Veves A, Murray HJ, Young MJ, Boulton AJM. The risk of foot ulceration in diabetic patients with high foot pressure: a prospective study. Diabetologia 1992; 35: 660-663.

17. Fernando DJ, Masson EA, Veves A, Boulton AJM. Relationship of limited joint mobility to abnormal foot pressures and diabetic foot ulceration. Diabetes Care 1991: 14: 8-11.
18. Pecoraro RE, Reiber GE, Burgess EM. Pathways to diabetic limb amputation: basis for prevention. Diabetes Care 1990; 13: 513521.

19. Boulton AJM. The diabetic foot. Med Clin N Am 1988; 72: 15I31530.

20. Young MJ, Cavanagh PR, Thomas G, et al. The effect of callus removal on dynamic plantar foot pressures in diabetic patients. Diabetic Med 1992; 9: 55-57.

21. Veves A, Masson EA, Fernando DJ, Boulton AJM. Use of experimental padded hosiery to reduce abnormal foot pressures in diabetic neuropathy. Diabetes Care 1989; 12: 653-655.

22. Veves A, Masson EA, Fernando DJ, Boulton AJM. Studies of experimental hosiery in diabetic neuropathic patients with high foot pressures. Diabetic Med 1990; 7: 324-326.

23. Boulton AJM, Franks CI, Betts RP, Duckworth T, Ward JD. Reduction of abnormal foot pressures in diabetic neuropathy using a new polymer insole material. Diabetes Care 1984; 7: 4246.

24. Boulton AJM, Bowker JN, Gadia M, et al. Use of plaster casts in the management of diabetic neuropathic foot ulcers. Diabetic Care 1986; 9: 149-152. 